Gynäkologe 2019·52:568-569

https://doi.org/10.1007/s00129-019-4456-0

(c) Springer Medizin Verlag GmbH, ein Teil von Springer Nature 2019

\author{
Tanja Fehm ${ }^{1} \cdot$ Peter Mallmann ${ }^{2}$ \\ 'Klinik für Frauenheilkunde und Geburtshilfe, Universitätsfrauenklinik Düsseldorf, Düsseldorf, \\ Deutschland \\ ${ }^{2}$ Klinik und Poliklinik für Frauenheilkunde und Geburtshilfe, Universitätsfrauenklinik Köln, Köln, \\ Deutschland
}

\title{
S3-Leitlinie Zervixkarzinom
}

Die Krebsfrüherkennungsuntersuchung mittels zytologischem Abstrich (KFU) hat wesentlich zur Senkung der Inzidenz von Zervixkarzinomen beigetragen [1]. Dennoch erkranken weiterhin ca. 4540 Frauen jedes Jahr an einem Zervixkarzinom [2]. Gründe sind in der fehlenden Teilnahme an der KFU (nur ca. 50\%) zu suchen und, in seltenen Fällen, in „PAPAbstrich-Versagern“ vor allem bei endozervikalen Zervixkarzinomwachstum.

In den Frühstadien ist in der Regel ein operatives Vorgehen indiziert, die Standardtherapie stellt die radikale Hysterektomie dar. Aufgrund der hohen Morbidität und des Wissens, dass die Parametrien nur selten befallen sind, sind derzeit operative Studienkonzepte wie das SHAPETrial initiiert worden, welche die onkologische Sicherheit der einfachen Hysterektomie im Vergleich mit der der radikalen Hysterektomie bei Tumoren unter $2 \mathrm{~cm}$ überprüfen. Im Stadium FIGO (Fédération Internationale de Gynécologie et d'Obstétrique) IaI gilt die Konisation oder einfache Hysterektomie ohnehin als onkologisch sicher. Die Implementierung der laparoskopischen Sentinellymphknotenbiopsie bei Tumoren bis $\mathrm{zu} 2 \mathrm{~cm}$ wird ebenfalls zur Reduktion der operativen Morbidität e.g. Lymphödem beitragen.

\section{》) Nach dem LACC-Trial bestehen Zweifel an der onkologischen Sicherheit des laparoskopischen Vorgehens}

Seit der Publikation des LACC-Trial sind allerdings Zweifel entstanden, ob das laparoskopische Vorgehen bei der radika- len Hysterektomie die gleiche onkologische Sicherheit aufweist wie das offene Vorgehen. Prof. Hillemanns wird in seinem Beitrag insbesondere diese Thematik beleuchten.

Meist ist in den frühen Stadien bei jungen Patientinnen der mögliche Fertilitätserhalt eine wichtige zusätzliche Fragestellung. Neben der Konisation besteht mit der Trachelektomie, die eine Resektion der Parametrien beinhaltet, eine weitere operative Option. Jedoch müssen diese Patientinnen ausführlich über die späteren geburtshilflichen Risiken aufgeklärt werden. Prof. Fehm et al. werden die unterschiedlichen Möglichkeiten je nach Tumorstadium und Vorhandensein von Risikofaktoren unter Berücksichtigung der aktuellen Leitlinien vorstellen.

Beim Stadium III ist die primäre Radiochemotherapie schon seit Jahren routinemäßig etabliert. Darüber hinaus wird mittlerweile auch im Stadium IIb die primäre Radiochemotherapie bevorzugt bzw. in der S3-Leitlinie Zervixkarzinom empfohlen. Ein operatives Lymphknotenstaging ist trotz geplanter Strahlentherapie zur Festlegung des Strahlentherapiefeldes indiziert, da die bildgebenden Verfahren einschließlich PET-CT (Positronenemissionstomographie-Computertomographie) eine geringere Sensitivität aufweisen. Die Kombination aus Radiochemotherapie und radikaler Hysterektomie sollte für die Patientinnen mit kurativem Ansatz vermieden werden. Daher sollte beim Vorliegen von einem bzw. mehreren Risikofaktor(en), e.g. N+, L1, V1, die eine Strahlentherapie notwendig machen, trotz frühen Tumorstadiums keine zusätzliche radikale Hysterektomie durchgeführt werden. Prof. Marnitz et al. 
werden uns die aktuellen Entwicklungen in der Strahlentherapie vorstellen.

Bei Patientinnen mit fortgeschrittenen Stadien wird derzeit die Rolle der neoadjuvanten Therapie untersucht, und die Bedeutung auf nachgeschaltete Therapiekonzepte wie die Strahlentherapie bzw. operative Therapie wird diskutiert. Möglicherweise könnten durch das Downstaging die Inzidenz befallener Lymphknoten und Parametrien reduziert und somit eine operative Therapie unter Verzicht auf eine Radiochemotherapie ermöglicht werden. Eine weitere Indikation ist gegeben bei der schwangere Zervixkarzinompatientin, bei welcher durch die Neoadjuvanz eine Verlängerung der Schwangerschaft zur Vermeidung einer extremen Frühgeburt ermöglicht werden soll. Dr. Stübs et al. wird das Thema der Neoadjuvanz in diesem Schwerpunktheft für uns beleuchten.

In den letzten Jahren waren die Behandlungsoptionen beim rezidivierten und metastasierten Zervixkarzinom mit Ausnahme der Chemotherapie sehr limitiert. Mittlerweile gibt es Hinweise darauf, dass innovative Therapien wie Checkpointinhibitoren ebenfalls von Nutzen sein könnten. Auch Studien mit therapeutischen HPV(humane Papillomaviren)-Vakzinierungen scheinen einen Fortschritt in der palliativen Therapie beim Zervixkarzinom zu bedeuten. Dr. Domröse et al. werden Ihnen daher die neuen therapeutischen Möglichkeiten vorstellen.

Wir wünschen Ihnen eine spannende Lektüre und verbleiben mit kollegialen Grüßen

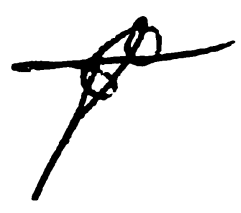

Prof. Tanja Fehm

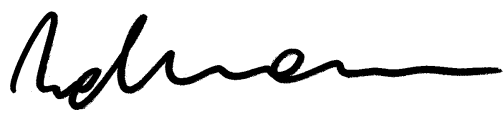

Prof. Peter Mallmann

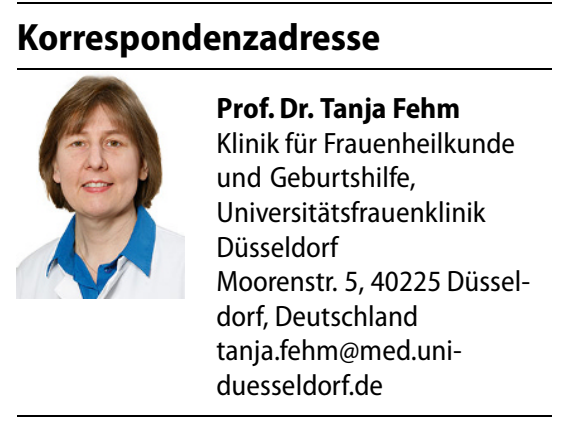

Interessenkonflikt. T. Fehm und P. Mallmann geben an, dass kein Interessenkonflikt besteht.

\section{Literatur}

1. https://www.krebsdaten.de/Krebs/DE/ Content/Krebsarten/Gebaermutterhalskrebs/ gebaermutterhalskrebs_node.html

2. Becker N (2003) Epidemiological aspects of cancer screening in Germany. J Cancer Res Clin Oncol 129(12):691-702

\section{Künstliche Intelligenz in der Medizin?}

Die Mehrheit der deutschen Bevölkerung steht Künstlicher Intelligenz (KI) in der Medizin offenbar positiv gegenüber.

$67 \%$ der deutschen Bevölkerung befürworten den Einsatz Künstlicher Intelligenz (KI) in der Medizin, beispielsweise im Bereich der Diagnostik. Das ergab eine repräsentative Umfrage des Meinungsforschungsinstituts Civey im Auftrag des Wissenschaftsjahres 2019 - Künstliche Intelligenz.

\section{Frage des Vertrauens}

Trotz der hohen Befürwortung von $\mathrm{KI}$ in der Medizin sehen die Bürger in Deutschland die Frage nach dem Vertrauen in KIgestützte Diagnosen differenziert. Rund $23 \%$ sagen, dass sie einer ärztlichen Diagnose "eindeutig mehr" oder "eher mehr" vertrauen würden, wenn diese auf Unterstützung einer $\mathrm{KI}$ zurückgreift. 35\% sind der Meinung, dass ihr Vertrauen nicht beeinflusst wird.

\section{Anonymisierte Gesundheitsdaten}

Bei der Bereitstellung von anonymisierten Gesundheitsdaten, die für einen effektiven KI-Einsatz in der Medizin notwendig sind, zeigen sich die Deutschen zwiegespalten: So spricht sich zwar eine Mehrheit von 57\% grundlegend dafür aus, Gesundheitsdaten anonymisiert bereitzustellen, wenn diese $\mathrm{KI}$-Diagnosen verbessern. Über $30 \%$ verneinen jedoch diese Bereitschaft.

Im Rahmen der neuen Diskussionsreihe „Karliczek. Impulse. Wie wir Künstliche Intelligenz nutzen wollen." tauschen sich Experten aus Wissenschaft und Praxis mit Bürgern über Chancen und Herausforderungen aktueller Aspekte der KI-Forschung aus. Die Wissenschaftsjahre sind eine Initiative des Bundesministeriums für Bildung und Forschung (BMBF).

Quelle: Bundesministerium für Bildung und Forschung, www.wissenschaftsjahr.de 